\title{
Introduction to the Minitrack on Digital Methods
}

\author{
Shawn Walker \\ Arizona State University \\ shawn.w@asu.edu
}

\author{
Alexander Halavais \\ Arizona State University \\ theprof@asu.edu
}

\author{
Matthew Weber \\ University of Minnesota \\ msw@umn.edu
}

\section{Introduction}

The Digital Methods minitrack focuses on addressing the methodological challenges of conducting research with digital and social media data. This is a new minitrack at HICSS, and was conceived as a forum to advance methodological discussions and to highlight innovations in digital research. While there has been an explosion of research using social media data to study human and machine behavior, the accompanying digital methods literature continues to lag. Researchers, practitioners, and students are left to adapt existing, "non-digital" methods or to reinvent methods via a process of trial and error. The papers presented in this minitrack highlight new approaches to digital methodology, and aim to detail specific methodological advances that can benefit the research community at large.

\section{Advancing digital methods in research}

There has been an explosion of research using social media data to study human behavior and social interaction in almost every domain of social science. While the body of literature using digital and social media data is growing at a staggering rate, accompanying methodological contributions about the process of conducting research with digital and social media data remains thin. The existing methodological literature is typically tool or technology driven, and not a result of empirical examination of the data collection process. This leaves researchers without an understanding of how to approach or evaluate the social media data collection process, and in turn, how to appropriately interpret findings from this type of research.

This minitrack addresses this gap by providing a venue to discuss methodological issues and approaches to conducting research with digital and social media data. We welcome papers related to methodological challenges for researchers including, but not limited to:
(1) the need for new methods for data collection and analysis, (2) adaptations of existing methods (3) issues of representation and sampling, (4) ephemerality of social media data, (5) holistic collection of digital social media data and associated content such as images/URLs/video, (6) preservation, archiving, and data sharing, and (7) impact of changing platform affordances, interfaces, designs, and APIs.

The three papers in the minitrack this year address the themes above and advance our understanding of the digital research methods and the ways in which researchers are studying emerging social media platforms such as TikTok. Together, the papers point to limitations of the current methods use to study digital platforms and online behavior - an area that is in need a innovation and development. The following sections will provide more detailed explanations of each paper.

\section{Does Your Smile Mean That You're Happy? - a Multi-Channel Analysis of Emotional Reactions}

The research developed by Eckhardt, Krönung, Reibenspiess, and Jaeger address the gap between selfreported measures of emotion and observational emotional coding systems such as Facial Action Coding System (FACS). Addressing these gaps in understanding of the measurement of emotion is important because a major focus of IS and digital research is the impact of emotion drivers on individual adoption decisions.

\section{Conducting Online Focus Groups - Practical Advice for Information Systems Researchers}

The second paper in this minitrack builds on the theme of advancing digital research methods. The paper by Schulze, Trenz, Cai, and Tan concretely address the nuts and bults of online focus groups as a research method - also addressing the issues, challenge, and 
approaches to mitigate them. The authors pay particular attention to the practicalities of recruitment, sampling, research setting, preparation, and facilitation. A review of the literature surrounding online focus groups is supplemented by the authors' own experiences with seven online focus groups in the context of digital platforms. Too often, the methodological issues in our fields of digital research gets hidden within the larger arc of publications or dropped entirely leaving a large gap in our understanding of digital methodologies.

\section{It's the Methodology For Me: A Systematic Review of Early Approaches to Studying TikTok}

The third paper in this minitrack by Kanthawala, Cotter, and Foyle analyzing the methods used to study the emerging social media platform TikTok. The authors examined journal articles and proceedings published using data from TikTok for this study. They found that studies rarely mention any ethical considerations of the collection and analysis of data from TikTok. Most studies in the authors' dataset use traditional forms of data collection which are not adapted to the unique affordances of the TikTok platform. The adaptation of existing methods and the development of appropriate methods for digital research still lags behind the research that is actually taking place - pointing to a large methodological gap in our fields.

\section{Future Directions for Digital Methods}

This year the Digital Methods minitrack provided a forum for starting new conversations about the development of methods that engage specifically with the challenges of conducting research in a digital media environment. In recent years, the increasing focus on a need to better understand human and machine behavior in online environments is increasingly critical to our knowledge of the world around us. We look forward to developing these conversations in future years as this minitrack continues. 